\title{
The acoustic emissions of cavitation bubbles in stretched vortices
}

\author{
Natasha A. Chang and Steven L. Ceccio \\ Department of Mechanical Engineering, University of Michigan, Ann Arbor, Michigan 48109
}

(Received 3 May 2010; revised 10 November 2010; accepted 17 November 2010)

\begin{abstract}
Pairs of unequal strength, counter-rotating vortices were produced in order to examine the inception, dynamics, and acoustic emission of cavitation bubbles in rapidly stretching vortices. The acoustic signatures of these cavitation bubbles were characterized during their inception, growth, and collapse. Growing and collapsing bubbles often produced a sharp, broadband, pop sound. The spectrum of these bubbles, and the peak resonant frequency can generally be related to quiescent flow bubble dynamics and corresponding resonant frequencies. However, some elongated cavitation bubbles produced a short tonal burst, or chirp, with frequencies on the order of a few kilohertz. Theses frequencies are too low to be related to resonant frequencies of a bubble in a quiescent flow. Instead, the frequency content of the acoustic signal during bubble inception and growth is related to the volumetric oscillations of the bubble while it interacted with vortical flow that surrounds the bubble (i.e., the resonant frequency of the vortex-bubble system). A relationship was determined between the observed peak frequency of the oscillations, the highly stretched vortex properties, and the water nuclei content. It was found that different cavitation spectra could relate to different flow and fluid properties and therefore would not scale in the same manner.
\end{abstract}

(C) 2011 Acoustical Society of America. [DOI: 10.1121/1.3626121]

PACS number(s): 43.30.Nb, 43.35.Ei [AJS]

Pages: 3209-3219

\section{INTRODUCTION}

In propulsors and turbo-machinery, the first form of cavitation is often vortex cavitation. Propulsor flows are typically characterized by concentrated regions of vorticity, where multiple co- and counter-rotating vortices are interacting together. Cavitation generally occurs in the core of these vortices since their static pressure is lower than that of the surrounding fluid. Predicting and scaling the onset of cavitation and its acoustic spectra is often important to the designers of such devices. Therefore, this topic has been studied extensively and a summary on vortex cavitation can be found in Arndt (2002). Generally, the primary (i.e., strongest) vortex in these flows is the first to incept, and scaling laws are based on this observation, such as that offered by McCormick (1962).

The conditions that generate primary vortex cavitation are predictable, but there exist flow conditions whereby weaker secondary vortices will incept before the primary vortex. This can occur when the core pressure of the secondary vortex is significantly reduced as the result of vortex stretching, which can result from its interaction with other, stronger vortices in the flow. Specifically, stretched secondary vortices can create localized regions of very low pressure in the vortex core. The drop in core pressure can amount to a significant level of tension, and given the availability of a nucleus, cavitation inception can occur, even at relatively high level of ambient static pressure. The prediction and scaling of such secondary vortex cavitation inception is more difficult due to three dimensional and unsteady nature of the secondary flow. See, for example, Chesnakas and Jessup (2003) for a propulsors flow, Gopalan et al. (1999) and Ran and Katz (1994) for jets, Belahadji et al. (1995), Iyer and Ceccio (2002), Katz and O'Hern (1986) and
O'Hern (1990) for shear layers, and Gopalan et al. (2002) for tip leakage vortex cavitation.

The dynamics and noise emission from vortex cavitation bubbles has been extensively considered (Arndt, 2002). Mellen (1954) showed that the acoustic intensity of cavitation decreases as $\mathrm{f}^{-2}$, and that the frequency at which this roll-off begins is related to the bubble size. Later studies related the observed cavitation spectra in a flow to single bubble dynamics in a quiescent fluid. In flow over a headform, Arakeri and Shanmuganathan (1985) observed that the acoustic intensity increases at higher frequencies $(>10 \mathrm{kHz})$ with the increase in the availability of nuclei, and that a peak can be observed forming at $63 \mathrm{kHz}$. In conditions of low cavitation single bubble dynamics was sufficient to relate the observed spectra, but with heavy cavitation and at high frequency this was not the case. Kumar and Brennen (1993) substantiate this result and showed that the Rayleigh-Plesset equation for bubble dynamics is not sufficient to predict the cavitation spectra at high frequency (30 to $80 \mathrm{kHz}$ ). Similarly, in propulsor flow Strasberg (1977), and Blake et al. (1977) attempt to scale the cavitation spectra based on single bubble dynamics and the size of the cavitation region. This scaling employed the assumption that all similar cavitating flows spectra can be related to basic flow properties, such as the local cavitation number and model size, and that all nuclei cavitate in the same manner. This method of estimating the vortex cavitation spectra works well for fully cavitating flows, but for incipient vortex cavitation it fails to predict resonant peaks of the spectra. Shen et al. (2002) apply a variation of this scaling method for a single bubble in a vortical flow, and validated their results to numerically simulated single incipient cavitation bubble spectrum that resembles a sharp broadband peak. 
A recent study of a ducted rotor propulsor at the U. S. Navy's Naval Surface Warfare Center - Carderock Division (Chesnakas and Jessup, 2003; Oweis et al., 2006a,b) shows that the location and inception pressure of the cavitation was associated with the presence of multiple, interacting vortices, and that a wide variation of spectra exist for the incepting vortex cavitation. Specifically, Chesnakas and Jessup (2003) found that the spectra of the incepting cavitation depended on the static pressure surrounding the propulsor. As the static pressure was lowered from a condition of no cavitation, the initial bubble acoustic signatures resemble a pop, a sharp broadband peak. As the pressure was further lowered the bubble signature took the form of a chirp. An acoustic chirp was much longer in duration than a pop, and it contained a welldefined tone when compared to the broadband pop. The measured tone of a chirp was between 2 and $6 \mathrm{kHz}$ in frequency and its persistence was of more than $2 \mathrm{~ms}$.

Cavitating jets present similar flow conditions where multiple vortices are interacting. Gopalan et al. (1999) conducted a study on cavitating jets where the primary and secondary vortices were characterized and the nuclei size and distribution were controlled to diameters between 150 and $200 \mu \mathrm{m}$. They observed cavitation events associated with the interaction of primary and secondary vortices with a circulation ratio of $\sim 0.1$. Long cylindrical bubbles would fill the core of the weaker secondary vortex, and the frequency of their acoustic signature would be the same as that of the resonant frequency of a spherical bubble of the same radius in quiescent flow and higher frequencies. The acoustical signatures observed were shorter than 1 to $2 \mathrm{~ms}$ in persistence (i.e., a pop). The frequency content of the bubble noise they measured was $29 \mathrm{kHz}$ or higher, even for cylindrical bubbles. Tonal bursts of noise were not reported. Similarly in a study of tip vortex leakage cavitation by Gopalan et al. (2002) report observing pop of the bandwidth of 20 to 28 $\mathrm{kHz}$ and 5 to $8 \mathrm{kHz}$ during bubble fission. Here again nuclei were supplied and of $\sim 100 \mu \mathrm{m}$ in size.

Predicting these varied incipient vortex cavitation spectra from non-cavitating flow properties has been extensively considered (Arndt, 2002), but few experimental studies have been able to connect the vortex properties, the bubble dynamics, and the resulting bubble acoustic emissions. Here, the dominant tone of the chirp bubble was experimentally related to the vortex properties, and it is shown to relate to the resonant frequency of the bubble vortex system. Generally the spectra predicted in the open literature were either the resonant spectra of a bubble in a quiescent flow or a broadband collapse. The simplest case is cavitation in a single line vortex, which was recently examined by Choi and Ceccio (2007). In that study a relatively large laser induced nucleus $(10-100 \mu \mathrm{m})$ was created in the core of a single line vortex, and the nucleus was then exposed to a pressure reduction and recovery as the vortex flowed through a Venturi. The resulting vortex cavitation bubble incepted immediately upon convecting into a region of fluid in tension in the core. The bubbles grew along the vorte $x$ axis, reached a maximum volume, and then collapsed when the pressure recovered. The noise produced was primarily associated with bubble collapse, and only pop type noise was recorded, while no chirp type bubbles were detected. Persistent acoustic or visual bubble oscillations were not observed during this series of experiments.

Numerical models of the cavitation phenomena and acoustic emission have also been developed. Latorre (1982) models a nuclei being captured by a line vortex and estimates its spectra using the Fitzpatrick-Strasberg formulation for modeling the bubble growth. This model fails to include the coupling between the vortex dynamics and the bubble dynamics (i.e., as the bubble grows the vortex is being displaced outward). Choi and Chahine (2004) develop a numerical model of tip vortex cavitation inception that also models a bubble being affected by the vortex pressure field, but not the effect of the bubble on the vortex. This study predicts that bubble fission would be louder than its initial growth and that its acoustic emission would take the form of a broadband pop. These models fail to predict the bubble chirps observed by Chesnaka and Jessup (2003).

Vortex cavitation inception resulting in a well defined tone of frequencies lower than the resonant frequency of just the bubble has been predicted analytically and numerically for cavitation bubbles in a line vortex by Choi et al. (2009). In this study the interactions between a single cylindrical bubble in the core of a line vortex and the surrounding vortical flow were computed, including the redistribution of the vorticity surrounding the bubble due to the volume changes of the bubble. It was found that bubbles could undergo radial oscillation, during bubble growth and collapse. These radial oscillations would take place with a period that was on the order of the vortex time scale $\tau_{v}=2 \phi r_{c} / u_{\theta \text {, max }}$, where $r_{c}$ and $u_{\theta, \max }$ are the (non-cavitating) core radius and maximum tangential velocity. Unlike previous studies where the bubble acoustic emission frequencies are related to the resonant frequencies of quiescent flow bubble, in this work it is related to the vortex/bubble flow system resonant frequency. It was also found that the amplitude of this oscillation was related to the timescale of the pressure reduction that initiated the cavitation, $\Delta T$, and the final core cavitation number, $\sigma_{c}$, once the minimum pressure was reached. As the core cavitation number decreases for a constant ratio of $\Delta T / \tau_{v}$, the amplitude of the radial oscillations increased. And for a constant core cavitation number, the amplitude of the oscillations increased as $\Delta T / \tau_{v}$ decreased.

Based on the numerical results of Choi et al. (2009), it is unlikely that a chirp producing cavitation event would be observed in the flow examined by Choi and Ceccio (2007). The 10-100 $\mu \mathrm{m}$ nucleus incepted immediately upon being subjected to a low value of tension in the core, and the timescale of the pressure reduction was relatively slow. Effectively, the vortex core cavitation number was between 0 and -0.9 and $\Delta T / \tau_{v}$ was greater than 1 . Under these conditions, it would not be expected that the vortex cavitation bubbles would undergo periodic radial oscillations that would produce an audible chirp. Similarly, Gopalan et al. (1999) had the nuclei sizes and distributions controlled to diameters between 150 and $200 \mu \mathrm{m}$. These bubbles would incept with minimal tension, so chirps would not be expected to be heard in this case with frequencies of $2 \mathrm{kHz}$ to $14 \mathrm{kHz}$ as predicted by Choi et al. (2009) based on the cavitating 
vortex time constant, $\tau_{v}$. In both studies, such harmonic tones were not reported.

Yet, chirp type acoustic emission from cavitation bubbles have been experimentally observed and reported by Chesnakas and Jessup (2003). The analysis of Choi et al. (2009) suggest that a vortex cavitation bubble will lead to a tonal pulse if a nucleus is exposed to a rapid application of strong tension, and this can take place in the core of a rapidly stretched vortex. A canonical flow that could lead to the flow condition described is a pair of counter-rotating parallel vortices (Devenport et al., 1997). In Chang (2007) it is shown that unstable pairs of counter-rotating vortices can provide a well-controlled flow field that can lead to rapid vortex stretching and cavitation of the secondary vortex.

In the present work, it is shown how vortex cavitation associated with the rapid vortex stretching can lead to both pop and chirp type acoustic emissions from incipient cavitation bubbles. Here, it is examined how the dynamics and acoustic emission of these bubbles relate to the underlying natural nuclei distribution and properties of the vortical flow. It is experimentally shown that the observed chirp frequency is related to the resonant frequency of the vortex/bubble flow system. This manuscript is divided into three additional sections. Section II will describe the experimental setup employed and the instruments used to characterize the flow field and cavitation. The acoustic emission of the vortex cavitation bubbles are related to the flow field in Sec. III, and conclusions are presented in Sec. IV.

\section{EXPERIMENTAL SETUP}

The experimental setup has two basic objectives: establishing a canonical flow whereby vortex cavitation can be studied, and studying the details of the acoustic signal generated by the bubbles in relation to the bubble dynamics and flow conditions. As stated in the introduction in real flow applications the spectra of these vortex cavitating bubbles can vary from broadband to narrowband (chirps). In canonical flows broadband cavitation bubbles have been studied extensively, but to the authors knowledge a canonical flows that generate chirp type vortex cavitation bubbles have not. From the literature, the form of cavitation of interest is formed with multiple interacting vortices (Chesnakas and Jessup, 2003; Oweis et al., 2006a,b) where weaker vortices were cavitating at higher cavitation numbers than the stronger vortices. Thus, the generation of a pair of parallel counter-rotating vortices was sought. The ratio of the circulation of this pair would be conducive to a rapid stretching of the secondary weaker vortex around the primary stronger vortex. The details of the flow set up are described in Chang (2007) and of the acoustic setup in Chang and Dowling (2009), and the following short description is given to aid the reader.

\section{A. Flow facility and test models}

The experiments were conducted in the University of Michigan 9-Inch Water Tunnel. Upstream of the test section the water tunnel has a series of flow management screens followed by a circular contraction with an area ratio of $6.4: 1$. The test section has a $22.9 \mathrm{~cm}$, or approximately 9 in. diameter inlet that smoothly transitions to a $22 \mathrm{~cm} \times 22 \mathrm{~cm}$ rounded rectangular cross section. The length of the test section is $1 \mathrm{~m}$. The test section has four acrylic windows that are $93.9 \mathrm{~cm} \times 10.0 \mathrm{~cm}$ for viewing purposes. The flow speed and static pressure in the test section can be controlled to values between 0 and $18 \mathrm{~m} \mathrm{~s}^{-1}$ and from near vacuum to $200 \mathrm{kPa}$. A de-aeration system is used to control the dissolved oxygen content of the water. The tunnel holds $3.8 \mathrm{~m}^{3}$ (1000 gal) of water, which is filtered at the inlet to $1 \mu \mathrm{m}$.

The vortices were generated with a pair of non-standard but similar hydrofoils. When mounted the camber was in the same direction. The hydrofoils were both $95 \mathrm{~mm}$ in span, with the first $167 \mathrm{~mm}$ in chord and the second $134 \mathrm{~mm}$ in chord. The smaller foil had a rounded edge. The two hydrofoils were mounted on opposite side windows at the same height and location along the length of the test section. The trailing edges of the two foils end at the same distance along the test section length (Fig. 1). The hydrofoil mounts allow rotation to achieve different angles of attack, creating counter-rotating vortex pairs with varying strengths. The spanwise gap between the two foils at zero angle of attack was $12.5 \mathrm{~mm}$.

The free-stream flow velocity, $U_{\infty}$, which was set to $10 \pm 0.3 \mathrm{~m} \mathrm{~s}^{-1}$ and the chord-based Reynolds number was $1.67 \times 10^{6}$ based on the larger foil. The foils and their angle of attack in conjunction with the free-stream flow velocity set the properties of the two vortices. These properties were measured at 1 chord-length downstream from the trailing edge of the foil once the vortex has rolled up and before the instability started which was approximately 1.5 to 2 chordlengths downstream of the trailing edge of the foil. The properties of the vortex were measured in a plane perpendicular to the free-stream flow with Stereo Planar Particle Imaging Velocimetry (SPIV) were a thousand double-pulsed uncorrelated images were averaged. The radial velocity distributions of the two vortices were then fit a Gaussian profile with a method similar to Choi and Ceccio (2007). The circulation and core size for the primary vortex were $-0.227 \mathrm{~m}^{2} / \mathrm{s}$ and $6.0 \mathrm{~mm}$, and for the secondary vortex $0.058 \mathrm{~m}^{2} / \mathrm{s}$ and 3.7 $\mathrm{mm}$. The distance between the two cores was $21.0 \mathrm{~mm}$. The error in these measurements is $\pm 6 \%$, and the details of the measurements are from Chang (2007).
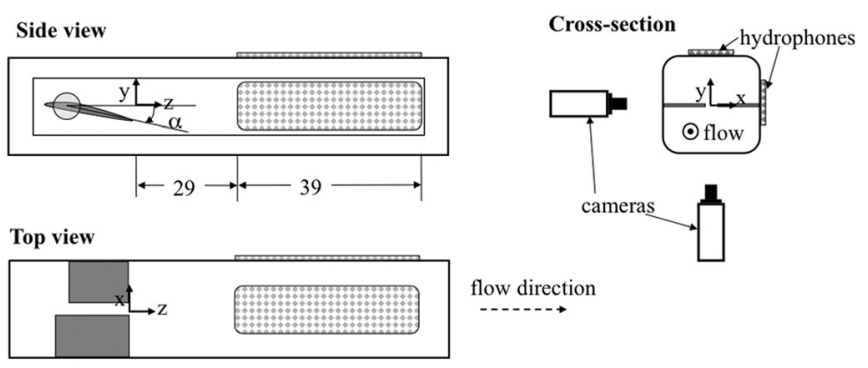

Location of hydrophones and focusing area for highspeed video camera

FIG. 1. Hydrophone and high-speed video camera set up. Dimensions are in centimeters. 
During the experiments, the water was de-aerated to achieve between $10 \%$ and $50 \%$ dissolved oxygen (DO) content at atmospheric pressure, which was measured with an Orion 810 dissolved oxygen meter. The free-stream static pressure, $P_{\infty}$, was varied to generate different cavitation numbers, and it ranged from 200 to $100 \mathrm{kPa}$. This corresponds to a free-stream cavitation number, $\sigma_{\infty}$, ranging from 4 to 2 , where $P_{V}$ is the water vapor pressure and $r$ is the water density:

$$
\sigma_{\infty}=\frac{P_{\infty}-P_{V}}{(1 / 2) \rho U_{\infty}^{2}} .
$$

The error of the cavitation number measured experimentally between different set ups and its true value is \pm 0.1 . The source of this error is the uncertainty in the free-stream velocity $( \pm 0.3 \mathrm{~m} / \mathrm{s})$ given the pressure transducer and temperature-thermocouple was accurate to $\pm 1 \mathrm{kPa}$ and $\pm 0.1^{\circ} \mathrm{C}$, respectively.

The presence of a nucleus is necessary for cavitation inception, and the size of the nucleus dictates the fluid tension required to initiate rapid volume growth. Hence, the observed event rate of the vortex cavitation, bubble dynamics, and the acoustics emission was strongly dependent on the free-stream nuclei content. The Cavitation Supceptibility Meter (CSM) from GEC Alsthom ACB was used to determine the nuclei density with varying dissolved oxygen content of the water in the tunnel water and that the water quality was consistent through different set ups. Detailed results can be found in Chang (2007). The CSM can produce a maximum tension in its Venturi of approximately $100 \mathrm{kPa}$. At a given tension cavitation inception is detected acoustically and the events are counted. The CSM then provides the number of nuclei activated for a given volume of water at a given pressure. The CSM was found to be accurate within $\pm 15 \%$ of the measured nuclei density for a given pressure. The inception pressure is then assumed to be the critical pressure, $P_{C R}$, of a clean gas nucleus with a bubble of radius $R_{C}$. As discussed in Brennen (1995) the critical pressure, $P_{C R}$, is related to a clean gas nucleus bubble radius, $R_{C}$, by

$$
R_{C}>\frac{4 S}{3\left(P_{V}-P_{C R}\right)},
$$

where $S$ is the surface tension and $P_{V}$ is the vapor pressure. The nuclei content of the water at different DO and pressures was measured, and it was found that at $25 \%$ DO most nuclei required a depression on the fluid pressure below vapor pressure of $100 \mathrm{kPa}$ or greater to incept, while at $50 \% \mathrm{DO}$ it required $50 \mathrm{kPa}$ of tension. These measurements imply that the majority of nuclei were of approximately $1-3 \mu \mathrm{m}$ in size and 5-8 $\mu \mathrm{m}$ in size, respectively. Given the error of the CSM the two nuclei density populations can still be considered distinct where the average size of the nuclei differs by a factor of 3 .

The flow and fluid setup described above ensured that the secondary, weaker, vortex would incept at a higher cavitation number than the primary, stronger, vortex. The inception location was downstream of the instability between 2 and 3 chordlengths downstream of the trailing edge of the hydrofoil.

\section{B. High speed video imaging and acoustic measurements}

Images of the vortex cavitation bubbles were acquired with two 8-bit Phantom V9.0 high-speed movie cameras with 50 or $85 \mathrm{~mm}$ focal length Nikon lenses in conjunction to $12 \mathrm{~mm}$ extension rings. The bubbles were illuminated from behind by four $300 \mathrm{~W}$ incandescent lights, and a light diffuser was used to prevent glare. The spatial resolution of the images varied depending on the effective resolution, frame rate, and the distance between the camera and the bubble being imaged. For gross bubble features and localization, the set up used yielded an image with $526 \times 1200$ pixels $(60$ $\mathrm{mm}$ across by $150 \mathrm{~mm}$ along the flow) or $504 \times 528$ pixels (approximately $45 \mathrm{~mm}$ square) with the respective frame rates of 2500 and 5400 frames per second (fps). The exposure time was varied depending on the lighting available to values between 180 and $31 \mu \mathrm{s}$. To generate a three-dimensional location of the bubbles imaged and observe their shape, the cameras were focused in the same area of the tunnel but at right angles, from the side and from below, as shown in Fig. 1. The movies were scaled by correlating the distance in pixels from the image of an immersed ruler at the approximate location of the bubbles. To study bubble dynamics, the cameras were set to acquire at 11000 frames per second of 526 pixels $\times 256$ pixels for a $33 \mathrm{~mm} \times 16 \mathrm{~mm}$ viewing area. At this resolution, the error associated with determining the location of the edge of the bubble within a frame was \pm 1 pixel or $\pm 0.06 \mathrm{~mm}$, therefore the error in determining the diameter of a bubble was $\pm 0.08 \mathrm{~mm}$.

An array of hydrophones was used to record the cavitating bubbles acoustic emission and locate the spatial position of sound-producing bubbles. The acoustic localization was conducted to confirm that the sound produced by the bubble that was high-speed video imaged corresponded to the acoustic recordings. A detailed description of the method and the array used can be found in Chang and Dowling (2009), with a summary provided here. The acoustic localization array was comprised of 16 hydrophones, Reson TC-4013. The receiving sensitivity of the hydrophones is $-211 \pm 3 \mathrm{~dB}$ re 1 $\mathrm{V} \mu \mathrm{Pa}^{-1}$ from $1 \mathrm{~Hz}$ to $170 \mathrm{kHz}$. The hydrophones were driven by a Reson VP-2000 voltage preamplifier that was powered by a Hewlett-Packard E3610A DC power supply at $12 \mathrm{~V}$ DC. To eliminate the $60 \mathrm{~Hz}$ noise signal from the power supply, the preamplifier filter was set to $100 \mathrm{~Hz}$ high pass and $1 \mathrm{MHz}$ low pass, and its gain set to $10 \mathrm{~dB}$. The signal from the preamplifier is then filtered with a Khron-Hite 3364 fourpole tuneable active filter. The filter setting used was Butterworth high pass $1 \mathrm{kHz}$, and low pass $200 \mathrm{kHz}$ with a $40 \mathrm{~dB}$ gain. Its attenuation is $24 \mathrm{~dB}$ per Octave. The hydrophones were mounted on the Lucite windows of the water tunnel test section. The Lucite window had a pocket of quiescent de-aerated water where the transducers were placed separate from the main flow by a $1 \mathrm{~cm}$ thick Lucite plate. The cavitation events of interest were occurring in the downstream half of the water tunnel test-section. Therefore, the receivers were placed in that half, with eight on the top and eight on the side windows ranging from 31 to $67.5 \mathrm{~cm}$ (1.8 to 4 chord lengths) from the hydrofoil trailing edge. Figure 1 shows the general 


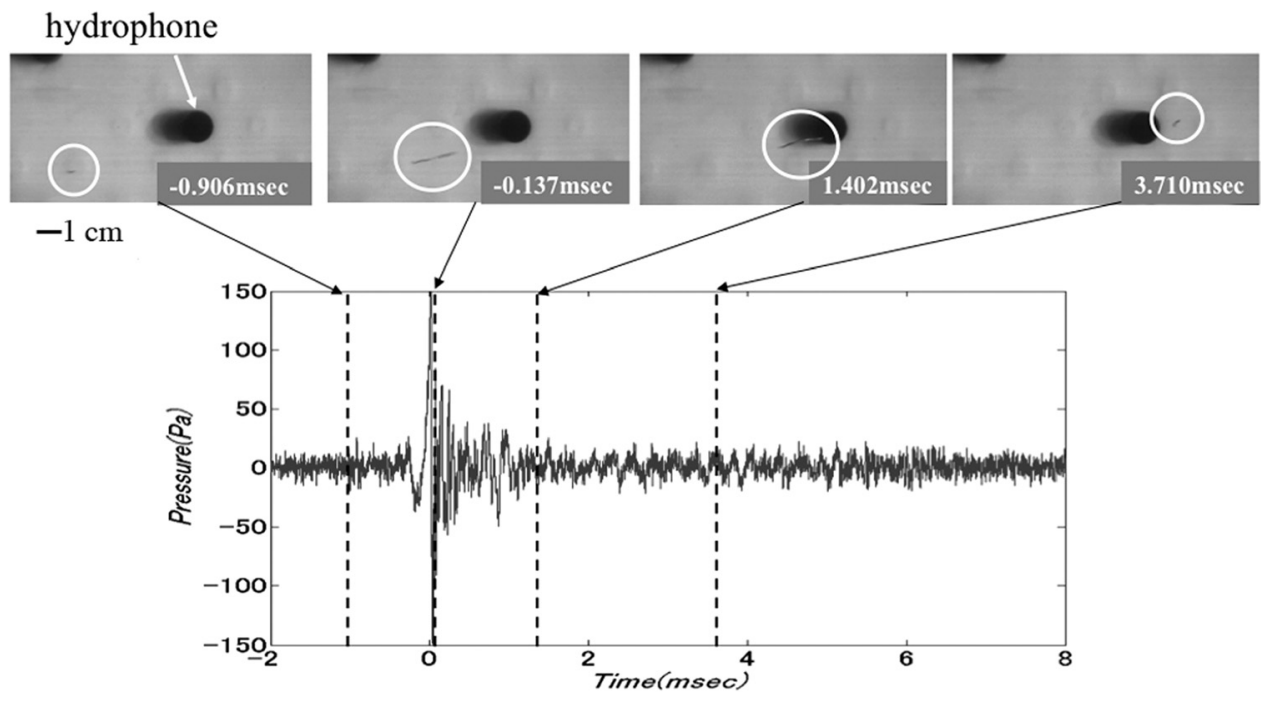

FIG. 2. Four images of a growing vortex cavitation bubble in the secondary vortex producing an acoustic pop. The acoustic emission recorded by a hydrophone is also shown. The image field-of-view is $78 \mathrm{~mm} \times 39$ $\mathrm{mm}$. Flow is from left to right. $\sigma_{\infty}=3.1$ and $\mathrm{DO}=25 \%$.

locations of the hydrophones. The signals from the acoustic array where then digitized by four National Instrument A/D systems, NI-PCI-6110 S, acquiring data synchronously at 1 $\mathrm{MHz}$. The sampling rate ensured that the Nyquist anti-aliasing criteria are met. The dynamic range of the $\mathrm{A} / \mathrm{D}$ cards is 12 bits for $\pm 10 \mathrm{~V}$.

The acoustic and video data acquired is correlated in time. A common trigger, the acoustic signal of one of the receiving hydrophones, provided the synchronization of these two independent systems. The trigger event was a $1 \mathrm{~V}$ rising signal, which then prompted the video and acoustic systems to save their respective buffers. This data could then be used to validate that the acoustic recording corresponds to the video of the bubble and compare the bubble dynamics with the sound it produces.

\section{ACOUSTIC EMISSION OF THE VORTEX CAVITATION BUBBLES}

\section{A. Acoustic emission from the incipient vortex cavitation bubbles}

The acoustic signal produced by the incipient cavitation was studied at dissolved oxygen contents less than $25 \%$ and high static pressure $157 \mathrm{kPa}\left(\sigma_{\infty}=3.1\right)$. The sound pulse was synchronously recorded with high-speed video to relate the acoustic signal to the inception, growth, and collapse of the bubble. The recorded acoustic signals could be sorted into two broad categories: pops which corresponded to a sharp noise pulse of duration less than $2 \mathrm{~ms}$, and a chirp corresponded to a periodic sound emission that lasted over 2 ms. These acoustic signal types could then be related to the state of the bubble when it was emitted (e.g., inception, growth, diminution, or collapse). For example, Fig. 2 shows a case where inception led to a pop. Figure 3 shows a collapsing bubble that produced a pop. However, in many cases, both growth and collapse did not lead to a detectable sound. Figure 4 shows two bubbles with the case where the growth and diminution of the bubble produced a periodic chirp, though the highest peak of the signal was during bubble growth. Here, 153 cavitation events were studied acoustically and with high-speed video. The acoustic signal was strongest during inception pops $(42 \%)$ and growth chirps (48\%) comprising $90 \%$ of the events. Collapse pops accounted for the remaining $10 \%$. Moreover, it was found that for the chirp growth/diminution bubbles, approximately $20 \%$ of bubbles produced the strongest portion of their

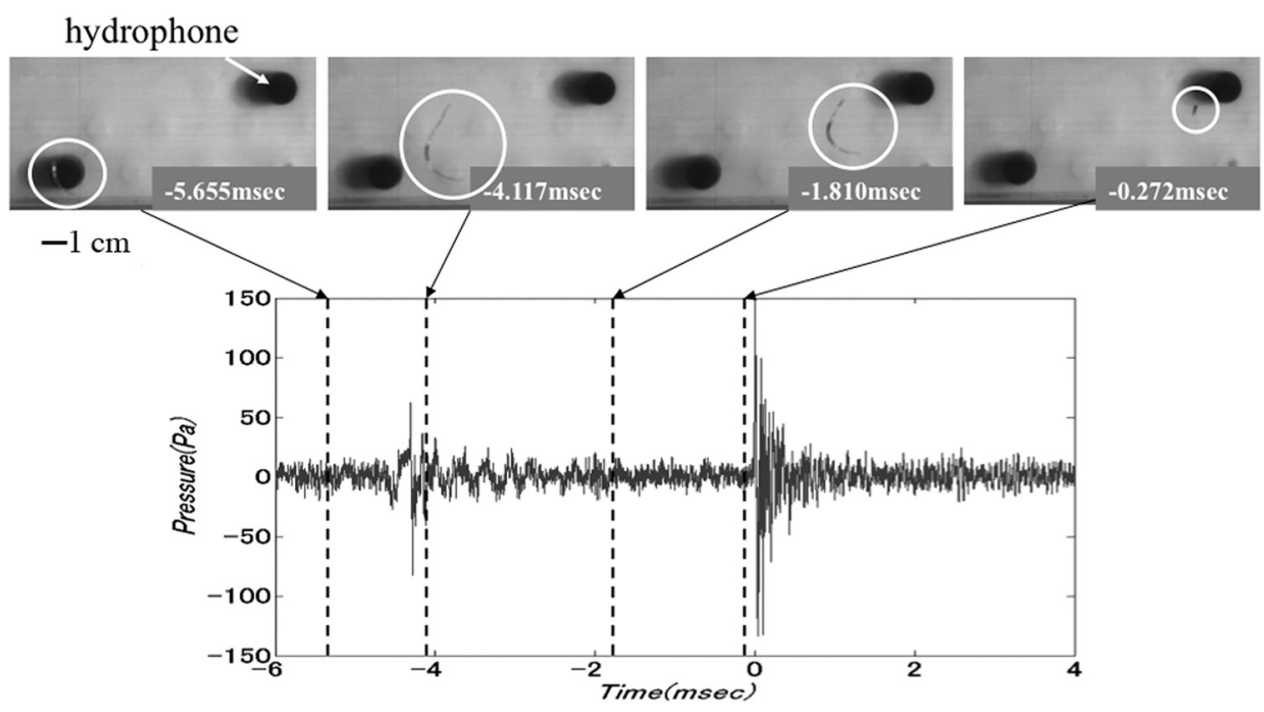

FIG. 3. Four images of a growing vortex cavitation bubble in the secondary vortex producing an acoustic pop during collapse. The broadband acoustic pulse was abrupt lasting approximately $1 \mathrm{~ms}$. The image field-of-view is $78 \mathrm{~mm} \times 39 \mathrm{~mm}$. Flow is from left to right. $\sigma_{\infty}=3.1$ and $\mathrm{DO}=25 \%$. 


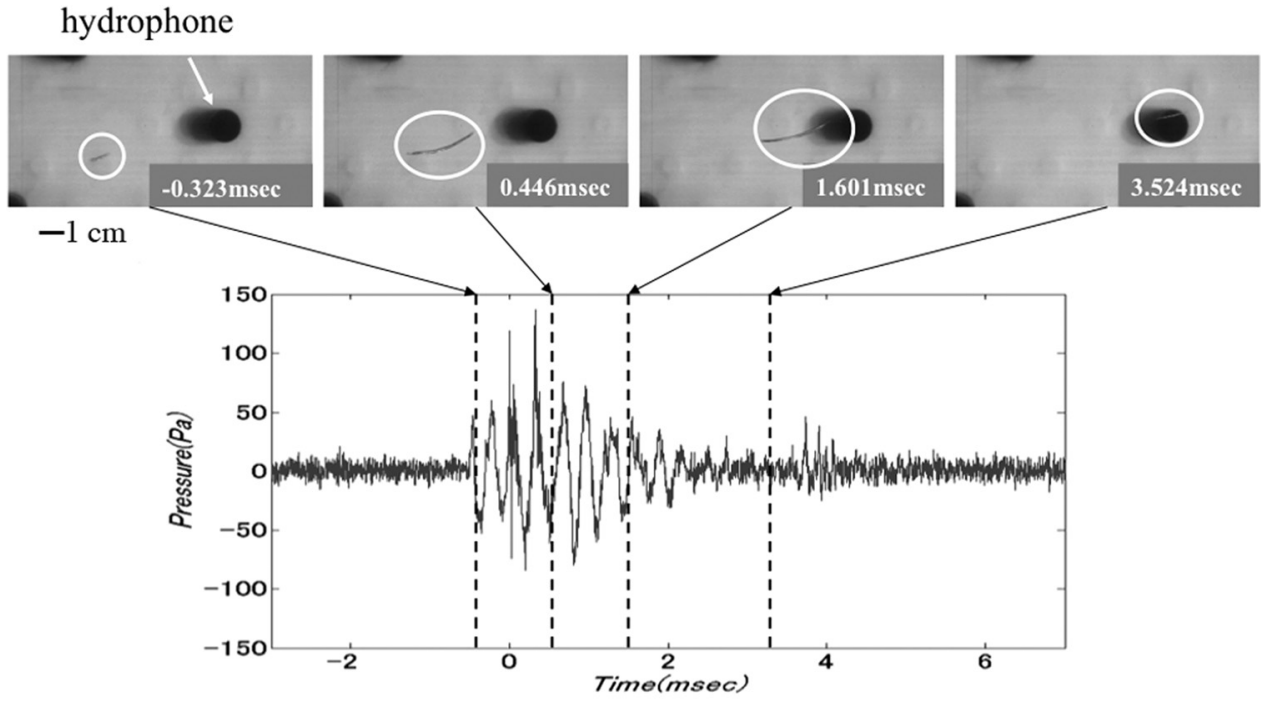

(a)
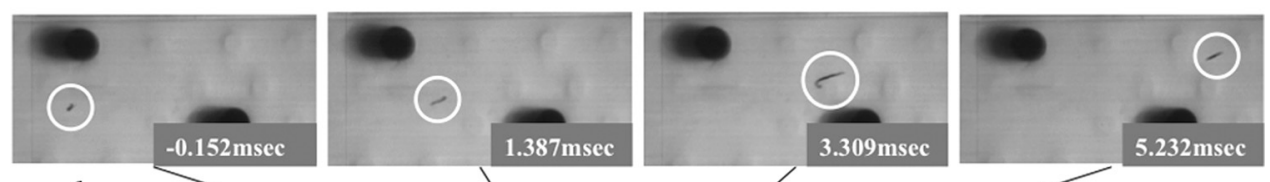

$-1 \mathrm{~cm}$

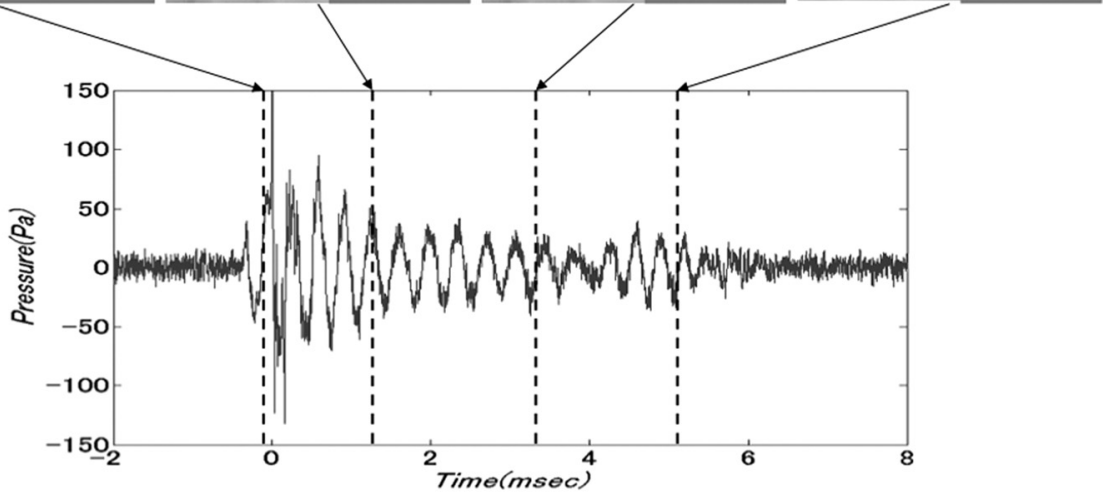

FIG. 4. Two sets of four images showing cavitation bubbles in the secondary vortex producing an acoustic chirp. The two bubbles produced acoustic pulses with a center frequency of $\sim 3$ to $4 \mathrm{kHz}$. The acoustic signal persisted from 5 to $10 \mathrm{~ms}$. The image area is 78 $\mathrm{mm} \times 39 \mathrm{~mm}$. Flow is from left to right. $\sigma_{\infty}=3.1$ and $\mathrm{DO}=25 \%$.

(b)

signature when the bubble was arresting its growth and initiating its diminution (Fig. 5). The acoustic traces of the pops and chirps were qualitatively similar to those observed by Chesnakas and Jessup (2003) for the cavitation occurring in the tip region of a ducted rotor.

The sound pressure level was estimated for the 153 bubbles measured and categorized into the different events. The acoustic data was sampled by using a taper-to-constant duration Tukey window that was 300 points for a growth pop or collapse, or 2000 points for a chirp. The sample data was transformed with a $2^{12}$ point Fast Fourier Transform (FFT) with additional zero padding as required. The recorded levels were corrected to account for the relative distance between the source and the receiver, in this case the closest hydrophone to the event. The reference pressure was $1 \mu \mathrm{Pa}$.

Figure 6 shows the resulting sound pressure level. The acoustic energy was concentrated at frequencies between 1 and $30 \mathrm{kHz}$ for a growth pop such as those recorded at inception, and between 2 and $6 \mathrm{kHz}$ during bubble chirp, growth/diminution, with a sound pressure level peak at approximately $3 \mathrm{kHz}$. The measured bubble collapse pop acoustic signal displayed a greater bandwidth than signals measured during growth. The growth pop and collapse pop show great overlap with the maximum sound pressure level value was measured at $\sim 10 \mathrm{kHz}$, though the collapse pop was found to be $\sim 2 \mathrm{~dB}$ lower than the growth pop at this peak value. From $100 \mathrm{~Hz}$ to $4 \mathrm{kHz}$ the collapse pop sound pressure level was higher than the growth pop. The acoustic emission of the cavitation bubbles was also related to the nuclei distribution. With higher dissolved oxygen content the nuclei are larger and more readily available. The resulting cavitation bubbles became more elongated, and their acoustic impulse during growth and collapse was weaker.

The resonant frequency of a spherical cavitation bubble can be estimated as discussed by Brennen (1995) with

$$
\omega=\left[\frac{3 \gamma P_{\infty}}{\rho R^{2}}+\frac{2 S(3 \gamma-1)}{\rho R^{3}}\right]^{1 / 2}
$$

where $\gamma=1$ is the polytropic gas constant, $S=0.071 \mathrm{~N} \mathrm{~m}^{-1}$ is the surface tension, $\rho=998 \mathrm{~kg} \mathrm{~m}^{-3}$ is the density of water, $P_{\infty}=157 \mathrm{kPa}, R$ is the bubble radius which is estimated from the videos to be at most $0.5 \mathrm{~mm}$ (with the 
(a)

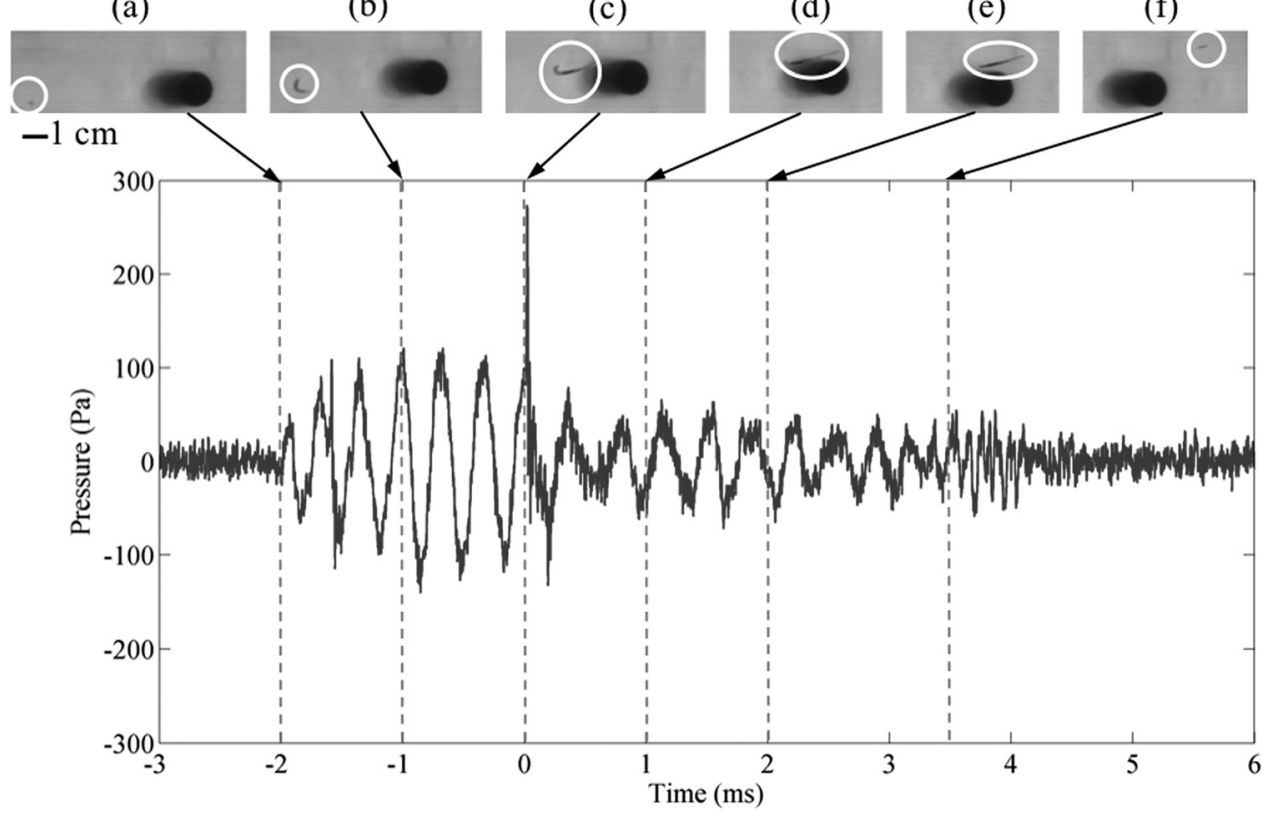

FIG. 5. Time trace of a cavitation bubble acoustical signature. (a)-(c) The inception process where the bubble slowly grows and chirps. The bubble experiences rapid growth and has a pop like signature, and then (c)-(e) the bubble is slowly shrinking and producing a chirp like signature. (f) Finally, the bubble collapses, and its collapse was quieter than the initial growth or pop. Flow is from left to right. $\sigma_{\infty}=3.1$ and $\mathrm{DO}=25 \%$. smallest bubble that could be resolved to be $\sim 0.2 \mathrm{~mm}$ ). The resonant frequency was found to be approximately 7 to 17 $\mathrm{kHz}$, which corresponds to the peak sound pressure level frequency value found for the bubble at the initial inception and final collapse pop. The peak sound pressure level frequency of a chirp is considerably lower, which could be due to the bubble length and persistence. However, Gopalan et al. (1999) found that the resonant frequencies of elongated vortex cavitation bubbles produced by secondary flows in a turbulent jet were of the same value or higher than that of a spherical bubble. The higher frequencies observed by Gopalan et al. (1999) could be due to the elongated bubbles fission and collapse to bubbles of smaller size than the radius of the elongated bubble or the incepting nucleus. Another

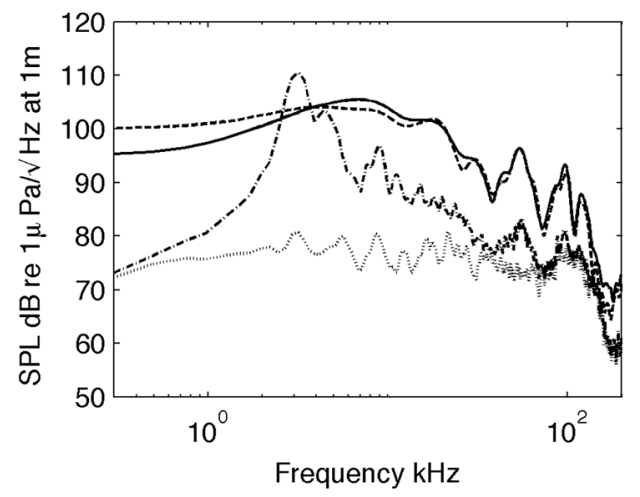

FIG. 6. Sound pressure levels of the distinct bubble dynamics and acoustic signature observed for the 153 counter-rotating vortex (CRV) bubbles: growth pop (solid line), collapse pop (dashed line), chirp (dash-dot line), and background noise (dotted line). $\sigma_{\infty}=3.1$ and $\mathrm{DO}=25 \%$. There were 119 growth pops (sometimes embedded in a chirp event) and 16 bubble collapse pops. There were also 65 bubbles that chirped some of which did not have a distinct time of rapid growth over which a pop could be measured. The acoustic signal was sampled with a taper-to-constant-duration ratio of 0.5 Tukey window of 300 points for the pops growth and collapse bubbles and 2000 points for the chirp events. The noise was similarly sampled to the chirps. A $2^{12}$-point Fast Fourier Transforms (FFTs) was conducted on the sampled data and zero padding was used as necessary. mechanism, such as coupling between the flow and the bubble dynamics, could be driving this lower resonant frequency in the chirp. This hypothesis will be discussed in more detail in the following section.

The strength of the acoustic signal is directly related to the volumetric acceleration of the bubble (Brennen, 1995)

$$
P_{A}=\frac{\ddot{Q} \rho}{4 \pi D}
$$

where $\ddot{Q}$ is the volumetric acceleration of the bubble, and $P_{A}$ is the estimated pressure at distance $D$ from source. Ceccio and Brennen (1991) and Kuhn de Chizelle et al. (1995) discuss how the rapid collapse of traveling bubble cavitation lead to sharp acoustic pulses that are very similar to the pop type signal observed in the present study. And, Choi and Ceccio (2007) recorded similar acoustic traces for collapsing vortex cavitation bubbles. Oweis et al. (2004) compared the sound produced by collapsing vortex cavitation bubbles to spherical bubbles with equivalent volume in an originally quiescent flow, and they found that when the bubbles remain nearly spherical in the core of the vortex, the noise producing efficiency of the bubbles approached that of the spherical bubbles. However, once the bubbles became significantly elongated, the intensity of the pop type sound produced upon collapse was reduced.

The non-dimensional acoustic impulse of the cavitation bubbles studied was estimated as in Choi and Ceccio (2007). The non-dimensional acoustic impulse is representative of the fraction of available potential mechanical energy that the bubble has converted to acoustic energy. It is defined as

$$
\hat{I}=\frac{I_{m} d_{H}}{\left[(1 / 3) \rho c \Delta P_{C} r_{b}^{3} \theta\right]^{1 / 2}},
$$

where $I_{m}$ is the integral of the peak pressure pulse, $\theta$ is the pulse peak width, $d_{H}$ is the distance between the bubble and 
the hydrophone, $\rho c$ is the characteristic acoustic impedance of water, $r_{b}$ is the bubble radius, and $\Delta P c$ is the collapsing pressure is generally estimated from the local cavitation number, $\sigma_{C}$. From Vogel and Lauterborn (1988), a sharp pressure pulse from a collapsing bubble has a measured pulse width of 10-100 ns that is beyond the resolution of the hydrophones used here. Therefore, it is assumed to be $10 \mathrm{~ns}$. Here, the local cavitation number could not be estimated directly from flow measurements, but as with $\Delta P c$ can be estimated based on the size of the nuclei incepted which were 1 to 10 micrometers. The bubble radius is estimated from the highest resolution high-speed videos taken as $\sim 0.3 \mathrm{~mm}$. In Fig. 7 the non-dimensional acoustic impulse of the 153 bubbles measured at a dissolved oxygen of $25 \%$ and a $\sigma_{\infty}=3.1$ and plotted in conjunction with the data from Choi and Ceccio (2007), the acoustic impulse of the inception and collapse pop were found to be of the same magnitude and a order of magnitude larger than those measured in the single line vortex experiment conducted by Choi and Ceccio (2007). In addition, the cases where the strongest portion of the signature occurs when the bubble was arresting its growth (and initiating its diminution), the emissions were also of the same magnitude as the inception and collapse pop (Fig. 5).

The explosive growth of a nucleus can produce large volumetric acceleration and, therefore, a detectable acoustic emission. However, the magnitude of inception and growth/diminution induced acoustic emission is often much less than the pulses produced upon final bubble collapse. This is illustrated in the scaling discussed by Blake et al. (1977). Observation of traveling bubble cavitation by Ceccio

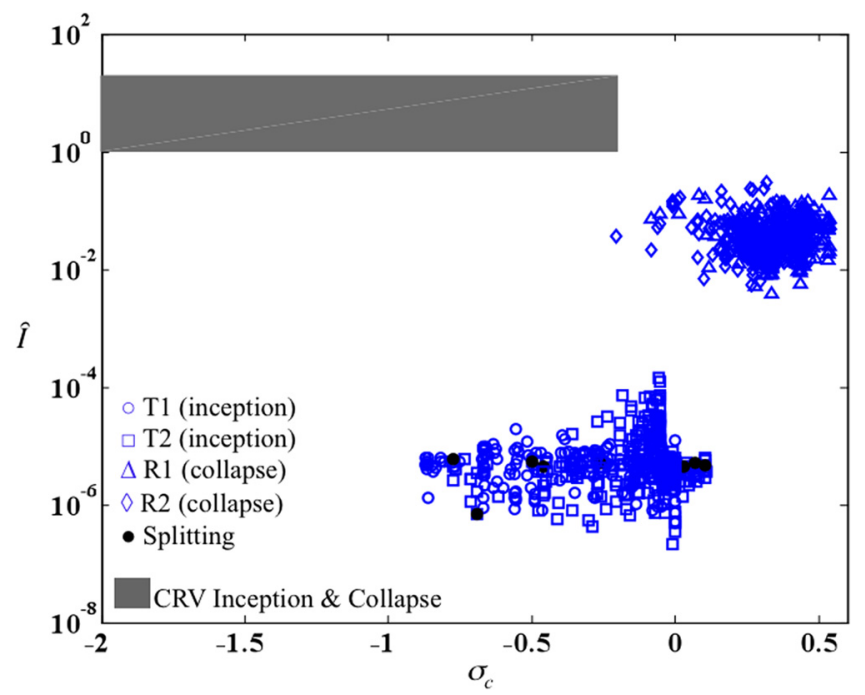

FIG. 7. (Color online) Figure reprinted from Choi and Ceccio (2007). The symbols T1, T2, R1, and R2 represent different single line vortices studied by Choi and Ceccio (2007) which had circulation ranging from 0.252 to $0.302 \mathrm{~m}^{2} / \mathrm{s}$ and core radius of $3.75-5.15 \mathrm{~mm}$. The grayed out area is where the non-dimensional acoustic impulse is estimated to be for the 153 counterrotating vortex cavitation bubbles with a $\sigma_{\infty}=3.1$ and $\mathrm{DO}=25 \%$. The bubble radius, $r_{b}$, is estimated for be $0.3 \mathrm{~mm}, \Delta P c$ is $\mathrm{O}\left(10^{-5} \mathrm{~Pa}\right)$, and $\theta=10 \mathrm{~ns}$ as in the Choi and Ceccio (2007). The cavitation number, $\sigma_{c}$, for vortex cavitation events in the present study were estimated from the original nuclei sizes $(1$ to $10 \mu \mathrm{m})$. If $\theta$ takes on the measured value, which ranged from $1.6 \times 10^{-5} \mathrm{~s}$ to $2.2 \times 10^{-4} \mathrm{~s}$, the values of $\hat{I}$ would then range from 0.02 to 0.23 . and Brennen (1991) also confirmed that the sound produced upon nucleus growth was usually much smaller than the collapse acoustic pulse, and that bubble deformations could mute the sound produced upon collapse. Moreover, Choi and Ceccio (2007) did not report significant acoustic emissions associated with bubble growth within their single vortex. These prior results appear at odds with the present observations in which substantial acoustic emission were detected upon vortex bubble inception and its growth/diminution. Only Gopalan et al. (2002) reports inceptions pop in their study of the effect of gap size in tip leakage vortex cavitation, which is similar to this case where a secondary flow incepts prior to the primary vortex.

An explanation for this apparent inconsistency comes from an examination of both the underlying cavitating vortical flow and the freestream nuclei distribution. The study of Choi and Ceccio (2007) employed relatively large (100 $\mu \mathrm{m}$ radius) laser-induced nuclei in the core of a single vortex. These large nuclei were then exposed to a relatively gradual pressure reduction as the vortex passed through a Venturi. Such large nuclei will cavitate when the local pressure just falls below the vapor pressure, and their rate of growth will be moderate as the surrounding pressure decreases. Similarly, Gopalan et al. (1999) nuclei of 150 to $200 \mu \mathrm{m}$ were supplied to the jet, again these nuclei would immediately cavitate upon experiencing tension. This is contrasted with the observations of vortex bubbles in the present flow, where the nuclei in the vortex cores were small, with equivalent radii of around $1 \mu \mathrm{m}$. These nuclei need a substantial tension, greater than $100 \mathrm{kPa}$, to incept. Such a strong tension can be achieved via vortex stretching, and bubbles exposed to the high tension will grow explosively, producing a rapid volumetric acceleration and, consequently, a strong acoustic pop, see Fig. 7. The rapidly varying pressure in the secondary vortex can also create situation where a sudden increase in pressure would arrest the growth of a bubble and lead to its diminution and eventual collapse. This would also produce a high volumetric acceleration and a pop acoustic emission, see Figs. 5 and 7.

\section{B. Chirping vortex cavitation bubbles and the inferred properties of the stretched secondary vortex}

Because a chirp is characterized by a periodic sound pulse consisting of many, lower frequency, pressure cycles, this type of acoustic emission (illustrated in Fig. 4) can be correlated to periodic oscillation of the bubble volume. A series of high-speed video images of chirping bubbles were taken at $11000 \mathrm{fps}$. The time series of images were used to estimate the time-varying bubble volume, and the resulting volumetric acceleration, after assuming that the bubbles were axisymmetric. The maximum duration of a given video recording was 2.5 milliseconds, and this, in conjunction with the frame rate, limited the volumetric oscillation frequencies that could be resolved from the video images to a range of 400-5500 Hz. This corresponds to acoustic wavelengths between 3.7 and $0.27 \mathrm{~m}$. Since the size of the bubble is less than one-tenth of the wavelength of interest, it can be assumed that the bubble behaves as a point source and that 

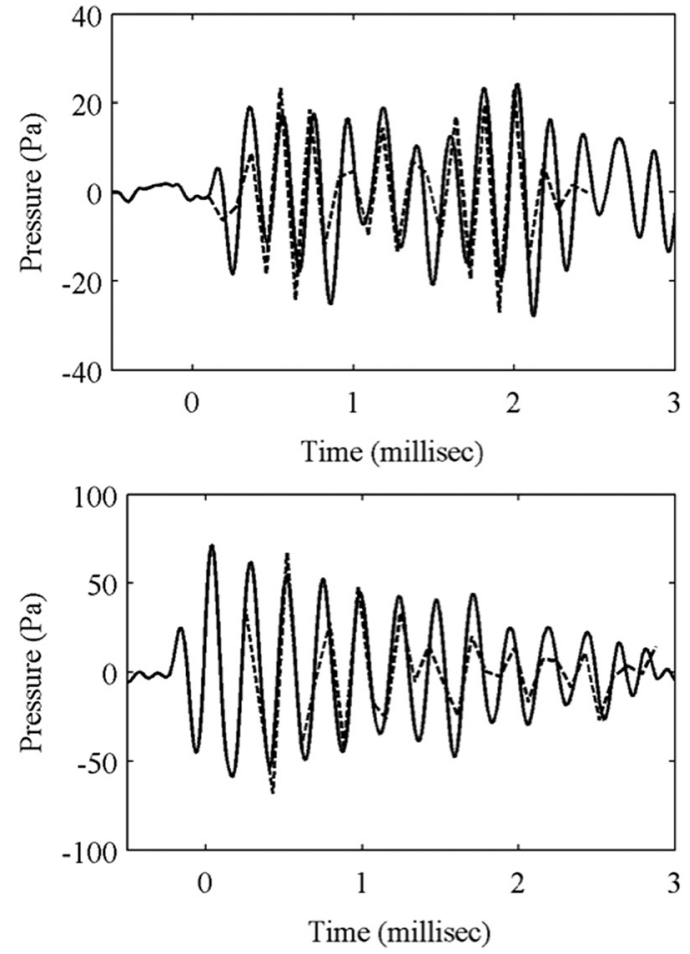

FIG. 8. The measured acoustic emission (solid line) of a single vortex cavitation bubble plotted with the computed pressure inferred from the volumetric acceleration of the visually recorded bubble (dashed line). The center frequency of the signal was $\sim 3-4 \mathrm{kHz}$. A correlation of (top) $84 \%$ and (bottom) $70 \%$ was found for these two cases. The phase of the signals were shifted with a lag corresponding to the maximum correlation point. There is an equivalent shift in the acoustic signal of (top) $-0.036 \mathrm{~ms}$ and (bottom) $-0.158 \mathrm{~ms} . \sigma_{\infty}=3.1$ and DO around $25 \%$.

the acoustic pressure can be estimated from the volumetric acceleration [Eq. (4)].

Figure 8 shows the measured and estimated acoustic emission from two chirping bubbles. Note that the acoustic signal was shifted in time with respect to the estimated pressure curve from the video data by a lag corresponding to the point of maximum correlation. The measured time lag is an artifact of a combination of the error in the synchronization of the two systems, where the video system at $11000 \mathrm{fps}$ could have delay with respect to the acoustic system of 0 to 0.09 milliseconds ( 0 to 1 frame period), and the relative difference between the speed of light and sound, which could generate a delay of the acoustic signal with respect to the video data of 0.04 to 0.18 milliseconds depending on the relative location of the source to the receiver. Thus, overall error in timing of the acoustic signal with respect to the video data was -0.050 to +0.180 milliseconds. The correlation between the two signals suggests that periodic volume oscillations of the bubble are, indeed, responsible for the chirp.

The single vortex cavitation bubbles studied by Choi and Ceccio (2007) did not exhibit any chirping type acoustic signature. Similarly, in Gopalan et al. (1999) or Gopalan et al. (2002) no chirp were reported, only pop type signals of $2 \mathrm{~ms}$ in duration. Once more, this difference can be ascribed to the variation in the nuclei populations and pressure histories in the vortex cores. In the present study, the high tension required for initiating bubble growth in the secondary vortex leads to explosive nuclei growth. In turn, as the bubble grows it will redistribute the surrounding vorticity, causing the local core pressure to increase, retarding the bubble growth. If this process occurs slowly, the volumetric oscillations of the bubble may not produce a detectable sound pulse. Conversely, the explosive growth can lead to strong interactions with the surrounding vortical flow and cause bubble oscillations. The numerical and analytical study of bubble dynamics by Choi et al. (2009) examined the volume oscillations of two- and three-dimensional vortex cavitation bubbles, and they discuss how the radial volume oscillations of vortex cavitation bubbles occur with a timescale related to the underlying vortex properties:

$$
\tau_{V}=\frac{2 \pi r_{C}}{u_{\theta, \max }}=\frac{4 \pi r_{C}^{2}}{\beta \Gamma_{0}}
$$

where $\beta=0.715$.

In the present experiment the chirping bubbles emit with frequencies on the order of $2.5-5 \mathrm{kHz}$. Following the discussion of the vortex dynamics of this flow that are presented in Chang (2007), a combination of a reduced secondary vortex core radius and increased axial jetting in the core can account for the observed inception at pressures between 112 and $157 \mathrm{kPa}$ (corresponding to $2.2<\sigma_{\infty}<3.1$ ). The initial value of the secondary core radius is $r_{C, S} \sim 3.5 \mathrm{~mm}$. And, with the assumption that the circulation is unchanged at $\Gamma_{S} \sim 0.06 \mathrm{~m}^{2} \mathrm{~s}^{-1}$, the stretching would have to reduce the core radius to $[1 / 2]$ to $[1 / 4]$ its original size to 0.9 $\mathrm{mm}<r_{C}<1.8 \mathrm{~mm}$ in order to produce the observed cavitation in the secondary vortex. Choi et al. (2009) showed how two-dimensional vortex cavitation had volume-oscillations periods, $T_{p}$, between $0.6 \tau_{V}<T_{p}<1.4 \tau_{V}$, while of a limited number of three-dimensional bubble simulations had periods on the order of $0.6 \tau_{V}$. For the stretched secondary vortex, the timescale of oscillations would then be expected to fall between $2.6 \times 10^{-4} \mathrm{~s}<\tau_{V}<10 \times 10^{-4} \mathrm{~s}$, implying that the frequency of the chirp can range between $0.7 \mathrm{kHz}$ $<1 / T_{p}<6.4 \mathrm{kHz}$. The measured frequencies of the experimentally observed chirps fall within this range.

Choi et al. (2009) showed that the oscillation frequency and amplitude depended on a number of flow parameters, including the original vortex circulation and core size, the core cavitation number, and the rate at which the core pressure was reduced. These parameters are not easily determined for the unstable vortical flow examined in the present study. Nevertheless, the measured volume oscillation period, $T_{p}$, was on the order of the inferred period of the stretched secondary vortex (e.g., $\sim 0.6$ to $1.4 t_{V}$ ). Hence, the measured sound frequency is that expected from the predicted secondary vortex properties. Finally, it should be noted the importance of the axial jet in depressing the pressure in the core and its relation to the acoustic signature. If the depression of the pressure in the vortex core were solely due to a reduction in core radius the bubble oscillation frequency would be increased. 


\section{Chirping vortex cavitation bubbles and nuclei content}

The type of acoustic emission of the cavitation bubbles was related to the nuclei distribution and free-stream cavitation number for the flow (Fig. 9). The fraction of bubbles that chirp was found to achieve a maximum value of $\sim 0.5$ for a dissolved oxygen content of $14 \%$ at $\sigma_{\infty}=2.5$. At higher or lower values of $\sigma_{\infty}$ the fraction was lower. However, to achieve $\sim 50 \%$ chirping bubbles at $44 \%$ dissolved oxygen content, $\sigma_{\infty}$ was increased to 3.6. In addition, it was observed for the $44 \%$ dissolved oxygen case that, as the number of chirping events increased to its $\sim 50 \%$ maximum, the period of the chirps changed in relation to the $\sigma_{\infty}$. At the lower $\sigma_{\infty}$, the periods of chirps tended to be longer, with some up to $1 \mathrm{~ms}$ long $\left(\sigma_{\infty}=2.2\right)$. As the $\sigma_{\infty}$ was increased, the average period of the chirp decreased, to a minimum observed value of $0.1 \mathrm{~ms}\left(\sigma_{\infty}=3.6\right)$.

The changes in the acoustic signature with $\sigma_{\infty}$ and dissolved oxygen content can be related to the results by Choi et al. (2009). The bubble and flow dynamics are coupled in vortex cavitation, though a cavitating bubble may not necessarily initiate an oscillation with frequency related to $\tau_{V}$. From the discussion in Brennen (1995) of Rayleigh-Plesset equation a smaller nucleus, requiring a lower critical pressure to be activated, will experience a greater volumetric growth once activated than a larger nucleus. Therefore, a small nucleus experiencing the same rate of pressure drop as a larger nucleus is more likely to overshoot its equilibrium bubble radius. At the higher dissolved oxygen content (i.e., larger nuclei) or at lower $\sigma_{\infty}$, (i.e., lower free-stream pressure), the tension needed to incept nuclei in the core of the secondary vortex is reduced. Therefore, these larger nuclei will grow slowly and may only produce a pop upon bubble growth or collapse. As the available nuclei in the flow become smaller in size (i.e., lower dissolve oxygen) or as $\sigma_{\infty}$ increases, the tension in the vortex core will have to be much higher to initiate inception.

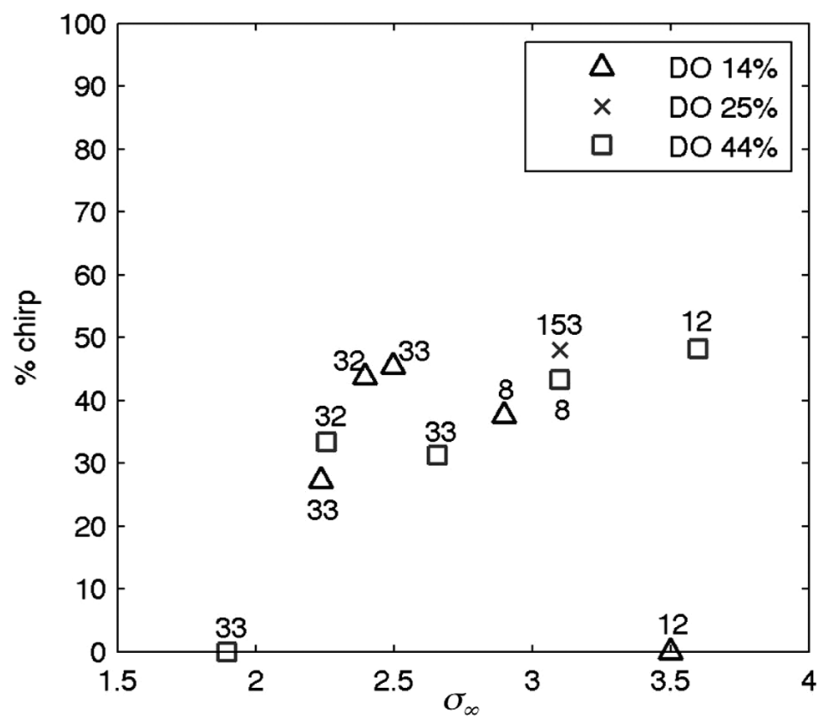

FIG. 9. Illustrated above is the percentage of events that produced a chirp for varying $\sigma_{\infty}$ and DO over a $3 \mathrm{~s}$ interval. Acoustic events were detected if the signal was $20 \mathrm{~dB}$ higher than the background noise level. The number by the symbol corresponds to the total number of cavitation events detected.
These smaller nuclei upon inception will experience greater radial acceleration than the larger nuclei and are likely to overshoot their equilibrium radius. This in turn initiates the radial oscillation of both the bubble and the vortex fluid surrounding it at a frequency related to $\tau_{V}$. Choi et al. (2009) showed that the greater the rate of change in pressure and/or the greater the absolute value of the pressure drop experienced by the nucleus, the greater will be the amplitude of oscillation of the bubble at a frequency related to $\tau_{V}$. Therefore, more chirping bubbles are expected for flows in which a rapid application of high tensions is needed to incept small nuclei. In fact, this trend is evident in Fig. 9 for $44 \%$ dissolved oxygen content at a $\sigma_{\infty}$ of 3.6 and for the $14 \%$ dissolved oxygen content at a $\sigma_{\infty}$ of 2.5 . In addition, since vortex stretching leads to the reduction in core pressure, it will also lead to a reduction in $\tau_{V}$ (due to smaller core radius). Therefore, the frequency of the chirp increases with increasing cavitation number, compared to the frequency observed from the similar vortex cavitation bubbles produced at lower cavitation number (i.e., lower required tensions produced via stretching), as stated above. Conversely, as $\sigma_{\infty}$ further increases and/or the dissolved oxygen content further decreases, the extent of the vortex stretching needed to initiate cavitation will be large enough to produce a rapid bubble growth, leading to a loud pop. And, such rapid bubble growth can disrupt the nominally two-dimensional vortical flow that originally surrounded the nucleus, which would preclude the establishment of radial oscillations of the bubble and vortex fluid. After inception, the bubble can quickly collapse and the majority of the events would not chirp. This is the case for 14\% DO content and $\sigma_{\infty}=3.5$, where from high-speed videos of incepting bubbles they were found to be short lived ( $\sim 2$ frames at $2500 \mathrm{fps}$ ).

\section{CONCLUSIONS}

The acoustic emission and dynamics of vortex cavitation bubbles produced from the unstable interaction of two counter-rotating vortices was examined and found to exhibit a variety of characteristics. Unlike the previous studies of cavitation in a single vortex (Choi and Ceccio, 2007), the low pressure in the cavitating vortex was produced after a weaker, secondary vortex was turned and stretched by the stronger, primary vortex. This process resulted in a sharp decrease in the core pressure of the secondary vortex, through a reduction in the core size and, possibly, the creation of an axial jetting flow in the vortex core (Chang, 2007). The reduced core pressure can reach tensions of order $100 \mathrm{kPa}$, which is sufficient to cavitate nuclei of order $1-3 \mu \mathrm{m}$ in diameter.

The cavitating nuclei often produced sharp popping sounds when they grew explosively, and when they collapsed. Explosive growth occurred when large tension surrounded the bubble at the point of inception, producing a sharp sound emission. This contrasts with the relatively gentle growth of the large laser induced nuclei cavitating in a line vortex studied by Choi and Ceccio (2007) where growing bubbles were not observed to make an audible sound pulse. Moreover, sharp transitions of core pressure, even surrounding an elongated bubble, would often produce an acoustic pulse resembling a pop in the present study. 
Elongated vortex cavitation bubbles were often observed to emit a periodic acoustic tone, or chirp, with an acoustic emission similar to that reported by Chesnakas and Jessup (2003). This second type sound was characterized by a periodic acoustic signal that was correlated to the growth and volume oscillation elongated bubble. Such acoustic signals were not observed in the study of Choi and Ceccio (2007), in the Gopalan et al. (1999) study of jets, or in the Gopalan et al. (2002). In those studies, the required fluid tension to initiate the vortex cavitation was much lower due to the larger nuclei $(\sim 100 \mu \mathrm{m}$ in size) undergoing inception. The resulting cavitation bubbles did not grow with rapid volume-acceleration, as compared to the bubbles studied here. Also, the spectrum of these bubbles was found to be related to the bubble's resonant frequency in a quiescent flow and unrelated to the vortex properties.

Volume oscillation of two-dimensional and elongated vortex cavitation bubbles were predicted analytically and numerically by Choi et al. (2009), and they found that elongated bubbles can undergo periodic volume oscillations under specific conditions that are unrelated to the resonant frequency of a bubble in a quiescent flow. The oscillation frequency is dependent on a number of flow parameters, including the rate at which the tension is applied to the original nucleus. Bubble volume oscillations resulted from the redistribution of the surrounding vorticity by the radial growth and collapse of the elongated bubble. The frequency of the volume oscillations scaled with the time-scale of the vortex, $\tau_{V}$. In the present study, elongated vortex cavitation bubbles were observed to emit chirps, and the acoustic signal was directly correlated to the measured volume oscillations of the elongated bubbles. The frequency of oscillation observed experimentally was within the range of frequencies expected from the inferred vortex properties of the stretched secondary vortex. Moreover, the propensity of bubbles to chirp was related to the underlying nuclei content and, therefore, the degree of tension needed to initiate inception.

Finally, the richness of the bubble dynamics and acoustics emissions of the vortex cavitation is indicative of the many flow parameters that play an important role in the bubble's inception, growth, and collapse. The type and frequency content of a bubble's noise emission is highly dependent on parameters such as the size of the nucleus, the level of tension required to initiate cavitation, and the rate at which the tension is applied and relieved. The present results illustrate how the effect of the available nuclei size, and their density can change the acoustic emissions of a secondary vortical flow.

\section{ACKNOWLEDGMENTS}

The Office of Naval Research supported this work under grant number N00014-03-1-0430, Dr. Ki-Han Kim, Program Manager. The authors would also like to acknowledge Dr. Jaehyug Choi and Dr. Ryo Yakushiji for their aid in collecting the data presented here.

Arakeri, V. H., and Shanmuganathan, V. (1985). "On the evidence for the effect of bubble interference on cavitation noise," J. Fluid Mech. 159, $131-150$.
Arndt, R. E. A. (2002). "Cavitation in vortical flow," Annu. Rev. Fluid Mech. 34, 143-175.

Belahadji, B., Franc, J. P., and Michel, J. M, (1995). "Cavitation in the rotational structures of a turbulent wake,” J. Fluid Mech. 287, 383-403.

Blake, W. K., Wolpert, M. J., and Geib, F. E. (1977). "Cavitation noise and inception as influenced by boundary-layer development on a hydrofoil," J. Fluid Mech. 80, 617.

Brennen, C. E. (1995). Cavitation and Bubble Dynamic (Oxford University Press, New York) pp. 65-91.

Ceccio, S. L., and Brennen, C. E. (1991). "Observations of the dynamics and acoustics of travelling bubble cavitation," J. Fluid Mech. 233, 633660.

Chang, N. A. (2007). "Acoustic characterization of cavitation in reverberant environments," Ph.D. dissertation, University of Michigan, Ann Arbor.

Chang, N. A., and Dowling, D. R. (2009). "Ray-based acoustic localization of cavitation in a highly reverberant environment," J. Acoust. Soc. Am. 125, 3088-3100.

Chesnakas, C., and Jessup, S. (2003). "Tip vortex induced cavitation on a ducted propulsor," in Proceedings of the 4th ASME-JSME Joint Fluids Engineering Conference, Honolulu, HI, FEDSM2003-45320.

Choi, J., and Ceccio, S. L. (2007). "Dynamics and noise emission of vortex cavitation bubbles," J. Fluid Mech. 575, 1-26.

Choi, J. K., and Chahine, G. L. (2004). "Noise due to extreme bubble deformation near inception of tip vortex cavitation," Phys. Fluids 16(7), 24112418.

Choi, J., Hsiao, C.-T., Chahine, G., and Ceccio, S. L. (2009). "Growth, oscillation, and collapse of vortex cavitation bubbles," J. Fluid Mech. 624, 255-279.

Devenport, W. J., Zsoldos, J. S., and Vogel, C. M. (1997). “The structure and development of a counter-rotating wing-tip vortex pair," J. Fluid Mech. 332, 71-104.

Gopalan, S., Katz, J., and Knio, O. (1999). "The flow structure in the near field of jets and its effect on cavitation inception," J. Fluid Mech. 398, 143.

Gopalan, S., Katz, J., and Liu, H. L. (2002). "Effect of gap size on tip leakage cavitation inception, associated noise and flow structure," Trans. ASME J. Fluids Eng. 124, 994-1004.

Iyer, C. O., and Ceccio, S. L. (2002). "The influence of developed cavitation on the flow of a turbulent shear layer," Phys. Fluids 14(10), 3414-3431.

Katz, J., and O'Hern, T. J. (1986). "Cavitation in large scale shear flow," ASME Trans. J. Fluids Eng. 108(3), 373-376.

Kuhn de Chizelle, Y., Ceccio, S. L., and Brennen, C. E. (1995). "Observations and scaling of travelling bubble cavitation," J. Fluid Mech. 293, 99-126.

Kumar, S., and Brennen, C. (1993). "A study of pressure pulses generated by traveling bubble cavitation,” J. Fluid Mech. 255, 541-564.

Latorre, R. (1982). "TVC noise envelope-an approach to tip vortex cavitation noise scaling,” J. Ship Res. 26, 65-75.

McCormick, B. W. (1962). "On cavitation produced by a vortex trailing from a lifting surface,” J. Basic Eng. 83, 369-379.

Mellen, R.H. (1954). "Ultrasonic spectrum of cavitation noise in water," J. Acoust. Soc. Am. 26(3), 356-360.

O'Hern, T. J. (1990). "An experimental investigation of turbulent shear flow cavitation,” J. Fluid Mech. 215, 365-391.

Oweis, G. F., Choi, J., and Ceccio, S. L. (2004). "Dynamics and noise emission of laser induced cavitation bubbles in a vortical flow field," J. Acoust. Soc. Am. 115(3), 1049-1058.

Oweis, G. F., Fry, D., Chesnakas, C. J., Jessup, S. D., and Ceccio, S. L. (2006a). "Development of a tip-leakage flow-Part 1: The flow over a range of Reynolds numbers," J. Fluids Eng. 128, 751-765.

Oweis, G. F., Fry, D., Chesnakas, C. J., Jessup, S.D., and Ceccio, S. L. (2006b). "Development of a tip-leakage flow-Part 2: Comparison between the ducted and un-ducted rotor," J. Fluids Eng. 128, 765-773.

Ran, B., and Katz, J. (1994). "Pressure fluctuations and their effect on cavitation inception within water jets," J. Fluid Mech. 262, 223-263.

Shen, Y. T., Chahine, G. L., Hsiao, C.-T., and Jessup, S. D. (2002). "Scaling of noise generation due to a single bubble in a cavitating trailing vortex," Report Number NSWCCD-50-TR-2002/053.

Strasberg, M. (1977). "Propeller cavitation noise after 35 years of study," Proceeding ASME Symposium on Noise and Fluids Engineering, Atlanta, GA, pp. 89-99.

Vogel, A., and Lauterborn, W. (1988). "Acoustic transient generation by laser-produced cavitation bubbles near solid boundaries," J. Acoust. Soc. Am. 84, 719-731. 